\title{
Correction to: Multi-objective hybrid genetic algorithm for task scheduling problem in cloud computing
}

\author{
Poria Pirozmand ${ }^{1}$ - Ali Asghar Rahmani Hosseinabadi ${ }^{2} \cdot$ Maedeh Farrokhzad $^{3} \cdot$ Mehdi Sadeghilalimi $^{2}$. \\ Seyedsaeid Mirkamali $^{4} \cdot$ Adam Slowik $^{5}$ (i)
}

Received: 25 November 2021 / Accepted: 25 November 2021 / Published online: 23 December 2021

(C) Springer-Verlag London Ltd., part of Springer Nature 2021

\section{Correction to: Neural Computing and Applications (2021) 33:13,075-3088 \\ https://doi.org/10.1007/s00521-021-06002-w}

Unfortunately, the article has been published with some errors in the reference citation.

The corrections are as follows:

In Page 13,077, under the heading Related works, in the seventh paragraph, the first line should read as,

"Alworafi [33] considered the load on resources and proposed a Hybrid-SJF-LJF (HSLJF) algorithm, which synthesizes the Shortest Job First (SJF) and Longest Job First (LJF) algorithms".

In Page 13,078 - In the first paragraph, the last line should read as "Simulation results showed a good improvement in makespan, execution time, and so on [38]".

Publisher's Note Springer Nature remains neutral with regard to jurisdictional claims in published maps and institutional affiliations.

The original article can be found online at https:// doi.org/10.1007/s00521-021-06002-w.

Adam Slowik

aslowik@ie.tu.koszalin.pl

Poria Pirozmand

poria@neusoft.edu.cn

Ali Asghar Rahmani Hosseinabadi

ark838@uregina.ca

Maedeh Farrokhzad

farrokhzad@gmail.com

Mehdi Sadeghilalimi

msv368@uregina.ca

Seyedsaeid Mirkamali

S.Mirkamali@pnu.ac.ir

1 School of Computer and Software, Dalian Neusoft University of Information, Dalian 116023, China

2 Department of Computer Science, University of Regina, Regina, Canada

3 Department of Computer Science, University of Science and Technology of Mazandaran, Behshahr, Iran

4 Department of Computer Engineering and IT, Payame Noor University (PNU), Tehran, Iran 